\title{
Atlantis
}

Critical Studies in Gender, Culture \& Social Justice

Études critiques sur le genre, la culture, et la justice

\section{Hear My Cry: Breaking the Code of Silence around Intimate Partner Violence among Black Women in and beyond Midlife}

\section{Ingrid Waldron, Eileigh Storey MacDougall and Lori E. Weeks}

Volume 42, Number 1, 2021

"Covid and the Academy" \& Open-themed Research

URI: https://id.erudit.org/iderudit/1082013ar

DOI: https://doi.org/10.7202/1082013ar

See table of contents

Publisher(s)

Mount Saint Vincent University

ISSN

1715-0698 (digital)

Explore this journal

Cite this article

Waldron, I., MacDougall, E. \& Weeks, L. (2021). Hear My Cry: Breaking the Code of Silence around Intimate Partner Violence among Black Women in and beyond Midlife. Atlantis, 42(1), 18-30. https://doi.org/10.7202/1082013ar

\section{Article abstract}

There is a knowledge gap about how the intersections of gender, race, culture, age, income, social class, and other identities shape Black women's experiences of intimate partner violence (IPV). In this qualitative study, we utilized an intersectional approach to examine how IPV is experienced and managed by racialized women, and in particular, our focus was to explore the IPV experiences of Black Nova Scotian women in and beyond midlife and their experiences of seeking support. Participant recruitment was predictably challenging, but we were able to collect in-depth interview data from a Black woman who identified as being in and beyond midlife and who had experienced IPV in the past and from three people who provided support to Black women in a paid capacity. An interpretive narrative approach was utilized to identify five dominant themes: descriptions of the experiences of IPV for Black women; strategies for coping with IPV; strategies in supporting Black women experiencing IPV; barriers in accessing support; and challenges in the delivery of support. The knowledge gained through this research provides important insights about the experiences, barriers faced, and how to address these challenges for Black women who experience IPV in and beyond midlife. (c) Ingrid Waldron, Eileigh Storey MacDougall, Lori E. Weeks, 2021

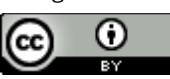

This document is protected by copyright law. Use of the services of Érudit (including reproduction) is subject to its terms and conditions, which can be viewed online.

https://apropos.erudit.org/en/users/policy-on-use/ 


\title{
Hear My Cry: Breaking the Code of Silence around Intimate Partner Violence among Black Women in and beyond Midlife
}

\author{
by Ingrid Waldron, Eileigh Storey-MacDougall, Lori E. Weeks
}

\begin{abstract}
There is a knowledge gap about how the intersections of gender, race, culture, age, income, social class, and other identities shape Black women's experiences of intimate partner violence (IPV). In this qualitative study, we utilized an intersectional approach to examine how IPV is experienced and managed by racialized women, and in particular, our focus was to explore the IPV experiences of Black Nova Scotian women in and beyond midlife and their experiences of seeking support. Participant recruitment was predictably challenging, but we were able to collect in-depth interview data from a Black woman who identified as being in and beyond midlife and who had experienced IPV in the past and from three people who provided support to Black women in a paid capacity. An interpretive narrative approach was utilized to identify five dominant themes: descriptions of the experiences of IPV for Black women; strategies for coping with IPV; strategies in supporting Black women experiencing IPV; barriers in accessing support; and challenges in the delivery of support. The knowledge gained through this research provides important insights about the experiences, barriers faced, and how to address these challenges for Black women who experience IPV in and beyond midlife.
\end{abstract}

Keywords: Black women; intimate partner violence; midlife; Nova Scotia; transformative justice

Acknowledgement: This research was supported by funding from the Justice Partnership and Innovation Program-Family Violence Initiative, Justice Canada.
Ingrid Waldron is Professor and HOPE Chair in Peace and Health in the Global Peace and Social Justice Program in the Department of History in the Faculty of Humanities at McMaster University. Her research explores the health and mental health impacts of inequality in Black, Indigenous, and other racialized communities. Her book, There's Something in the Water: Environmental Racism in Indigenous and Black Communities, was published in 2018 and was turned into a Netflix documentary in 2020 by actor Elliot Page. Her journal publications have explored Black women's experiences with mental illness and help-seeking, women's experiences managing low-income and poverty, and the traumatizing aftereffects of anti-Black police violence.

Eileigh Storey-MacDougall is a Social Worker in Halifax, Nova Scotia. After completing her Bachelor and Master of Social Work at Dalhousie University, she spent several years working with women and trans folks and their children who were experiencing homelessness. She has worked with older adults in both clinical and community settings, and acted as a board member for a registered charity dedicated to supporting older adults in the community. She has supported several research projects focused on the experiences of older adults. She continues to work as a Registered Social Worker (RSW) in Nova Scotia.

Lori E. Weeks a professor in the School of Nursing and holds a cross-appointment in Gender and Women's Studies at Dalhousie University. Lori has expertise in aging and family studies and uses quantitative and qualitative methods to examine care and support services for older adults and their caregivers and factors affecting the health of seniors. Her research often focuses on the needs of vulnerable older adults both in the community and in 
long-term care homes. She has conducted research on the abuse of older adults with particular focuses on supports for older women experiencing intimate partner violence. Lori is a member of the Abuse and Neglect of Older Adults Research Team in the Maritimes, a research team affiliated with the Muriel McQueen Fergusson Centre for Family Violence Research at the University of New Brunswick.

\section{Introduction}

There is a gap in our knowledge about how the intersections of gender, race, culture, age, income, social class, and other identities shape Black women's experiences of intimate partner violence (IPV). In this qualitative study, we used an intersectional narrative approach to explore the IPV experiences of Black women in and beyond midlife. As little is known about these women, we interviewed them and those who, in a paid or unpaid capacity, support them. The knowledge gained through this research provides important insights about the challenges for Black Nova Scotian women in their experiences of IPV and the barriers faced in getting support. We begin by providing an overview of our theoretical framework including an intersectional approach. This is followed by a review of the literature on Black women's experiences of IPV and in seeking support, and the limited knowledge about racialized women in and beyond midlife who experience IPV. We then provide details about the methods followed in conducting this study and the results. We conclude by highlighting our key findings in relation to the existing literature, the implications of our research for practice, and future research.

\section{Theoretical Framework}

In this paper, we are using an intersectional narrative approach to examine how race, culture, age, immigrant status, and class shape how IPV is experienced and managed by Black Nova Scotian women. Indeed, Black and other racialized women's location at the intersection of IPV and state-sanctioned racial and gender violence provides them with a unique understanding of and approach to dealing with their experiences of IPV. In this article, we use the term "racialized" to refer to nonWhite people who experience overt and/or systemic racism based on skin colour and phenotypic features, and we recognize that the process of racialization is one in which racial hierarchies are embedded within our social institutions in ways that determine the extent to which we are able to access various resources. Race developed over the years as a principle of social organization and identity formation and has been used to categorize, to include, and to exclude people based on physical traits. Systemic racism refers to how racial hierarchies become entrenched within our social system resulting in the unequal distribution of resources, such as the denial of access, participation, and equity to racialized people for basic needs, such as education, employment, and housing. 
It manifests in the policies, practices, and procedures within our systems that may directly or indirectly promote, sustain, or entrench differential advantage or privilege for people of certain races (Feagin, 2000; Miles, 1989). Therefore, as Hicken and colleagues (2018) observe, "Racialization is the process by which social meanings are attached to a constellation of biological, phenotypic, or otherwise observable features."

This paper also uses the concept of state-sanctioned racial and gendered violence (Waldron 2019) to expand the concept of state-sanctioned violence (AlimahomedWilson \& Williams 2016; Menjivar 2016; Pellow 2016) and pinpoint the specific ways in which government policies and decisions harm or otherwise disadvantage racialized women, preventing them from meeting their basic needs and rights related to employment, income, health care, and other resources (Gazso, McDaniel \& Waldron, 2016; Waldron \& Gazso, 2017; Waldron, 2019). Racialized women's experiences of state violence are specifically shaped by their location at the intersection of race and gender and, consequently, their exposure to gendered and racialized inequalities.

For Black women, trauma is specifically experienced through their exposure to race and gender-based violence, including the violence of homophobia and transphobia in their relationship to the state and social structures, and additionally in their intimate and other personal relationships. For example, Okeke-Ihejirika, Salami and Karimi (2019) found that African immigrant women in Alberta experience specific stressors impacting their mental health which are related to the challenges faced in transitioning and integrating into Canadian culture. Contributing to these stressors are the following: the absence of support that was present in their home countries, language barriers, the devaluation of their credentials, job inequalities, economic insecurity, lack of access to social networks, and changes to traditional gender roles which put these women at increased risk for IPV. Martin, Boadi, Fernandes, Watt, and Robinson-Wood (2013) found that Black women's experiences of depression are caused by systemic racism, micro-aggressions, poverty, cultural socialization, obesity, diabetes, and exposure to interpersonal and community violence. Bukowski, Hampton, Escobar-Viera, Sang, Chandler and Henderson (2019) found that the factors which were significantly correlated with symptoms of depression for Black transgender women (BTW) included IPV, physical and verbal violence, social support, gender identity, sex work, level of education, employment status, re- lationship status, and homelessness in the previous year. BTW were found to be at a higher risk for IPV resulting from their intersecting identities, limited economic opportunities due to discrimination and stigma, and financial dependency on their partner. For example, when racialized women who have been victims of IPV seek support from the criminal justice system and service providers, their concerns are often dismissed and they often report experiencing discrimination and cultural insensitivity (Bundy 2019; Tam et al. 2016). Traditional ways of supporting women who experience IPV do not acknowledge the adversities that contribute to racialized women's experiences of violence and oppression (Lacey et al. 2015; Long and Ullman 2016; Sabri et al. 2016).

Although there continues to be a gap in research on racialized women's experiences of IPV, studies have been emerging in recent years (Godoy-Ruiz, et al. 2015; Rezazadeh and Hoover 2018; Tam et al. 2016). Further research on the experiences of racialized women in Canada who suffer IPV is needed in order to inform culturally sensitive practices. The focus in this study is Black women's experiences of IPV, and we refer to sources focusing on Black women in the United States (African American women), Black women of Caribbean heritage residing in Canada, and Black women born in Africa who reside in Canada. We recognize that the cultural contexts within which these Black women experience and address IPV will be different given the differences in historical, political, social, and economic contexts between Canada and the United States. We argue, however, that these women's shared experiences of state-sanctioned racial and gender violence in these White-dominant societies provide a fundamental analytical entry point to highlight how race and gender intersect in ways that not only create barriers to help-seeking among Black women in White dominant societies (regardless of cultural context or residence in Canada or the United States), but also have implications for the considerations that need to be made in providing services that meet the needs of Black women.

\section{Women at the Crossroad: Black Wo- men's Experiences of IPV}

Researchers have examined how Black women who experience IPV are often examined as a monolith since the literature often fails to address the very different ethnic and cultural backgrounds and stories of Black women (Lacey et al. 2015). Since Black women experience mar- 
ginalization due to race, gender, and the feminization of poverty (Gazso and Waldron 2009; Gazso, MacDaniel and Waldron 2016; Waldron and Gazso 2017), an analysis that acknowledges Black women's intersectional identities is important if we are to understand their unique experiences of IPV, and the challenges they face accessing support. Black women's experiences can be characterized in terms of their structural location at the crossroad of race, gender, class, age, sexual orientation, and other social oppressions.

Also contributing to the challenges which Black women face reaching out for help is the "strong Black woman" trope-a cultural phenomenon that places unreasonable expectations on Black women to be nurturing and supportive figures in their families and communities, often resulting in the undermining or dismissal of Black women's vulnerability (Etowa et al. 2017; Waldron 2019; Waldron 2002). Etowa and colleagues (2017) observe that Black women in Eastern Canada felt a pressure to be the "backbone of the Black community" (383) by taking care of all family members, putting the needs of others before themselves, being self-sufficient and invulnerable, and being willing to sacrifice and take on limitless burdens.

The characterization of Black women as nurturing, strong, invulnerable, and resilient "superwomen" is deeply rooted, and it serves to undermine the racialized physical and mental trauma that Black women have long had to endure, often causing them to internalize this damaging stereotype, as well as minimize, ignore, or deny the many challenges they face, including IPV (hooks 1993; Schreiber, Stern \& Wilson 2000; Waldron 2002). Black women's internalization of the strong Black woman stereotype as a central component of Black womanhood can be harmful to their well-being because it promotes a perception of Black women as having excessive strength, caretaking abilities, and emotional restraint and, consequently, may be detrimental to their self-care behaviours, especially in the case of IPV where they may be less likely to seek support.

\section{Black Women Seeking Support for IPV}

Studies show that Black women are less likely to seek support for their experiences of IPV than White survivors and are often unaware of available resources (BentGoodley 2013; Sabri et al. 2015). St Vil and colleagues (2017) found that Black women utilized three types of survivor strategies: internal strategies of faith and reli- gion, interpersonal strategies of leaving the abuse, and external reliance on informal and/or formal supports. Many Black women reported relying on the church community and friends and family for support, while only some looked for support from organizations that specialized in supporting women who experience IPV (Bent-Goodley 2013; St Vil et al. 2017). Other studies indicate that although Black women are hesitant to use legal resources, they are more likely to access them when there is a high risk for lethality (Sabri et al. 2014). Compared to White women, Black women are also more likely to seek medical treatment due to the seriousness of IPV (Bent-Goodley 2013). Black women are also less likely to access counselling and other professional supports because of a fear of contributing to racist and negative stereotypes of Black men and Black communities as inherently dangerous (Al'Uqdah, Maxwell and Hill 2016; Sabri et al. 2015).

\section{Racialized Women in and beyond Mid- life and IPV}

In this article, we focus on older women. There is no consensus in IPV research on the specific age at which a woman is considered older than midlife. Typically, researchers include women who have at least reached the age of 50 years, which is sometimes described as midlife or older than midlife (Weeks and LeBlanc 2011). We believe that referring to women in and beyond midlife clarifies our population of interest as women who are not caring for dependent children. While our knowledge is far less complete on IPV among older versus younger women, mistaken assumptions are that woman of all ages experience IPV in similar ways, and that IPV ceases with old age (Hightower, Smith and Hightower, 2006). Prevalence studies show that between $15 \%$ and $30 \%$ of older women report IPV at some time over their life (Bonomi et al. 2007; Montero et al. 2013; Stöckl and Penhale 2015). In a Quebec study of coroners' files of homicide victims aged 65 and older reports that $89 \%$ of the victims were female, and of these, $93 \%$ were current or former spouses of male perpetrators (Bourget, Gagné and Whitehurst 2010). It is recognized that like other types of abuse, IPV among older women is under-estimated.

Research examining IPV among older women from various racialized groups is very limited (Mcgarry, Simpson and Hinchliff-Smith 2011; Pathak, Dhairyawan and Tariq 2019). Most studies include ethnically homogen- 
eous samples of predominantly White participants or they include more diverse participants, but the results are not presented by racialized groups (Sormanti and Shibusawa 2008). As abused women may have varying needs based on race and ethnicity, understanding the needs of specific groups of women is important (Roberto, McPherson and Brossoie 2013), including women in and beyond midlife. Additional research is needed that focuses on the service needs for older racialized women experiencing IPV.

\section{Methods}

This study was conducted as part of a larger study focused on identifying and responding to the needs of diverse older women who experience IPV (Weeks et al. 2021). The main purpose of the larger study was to gain insights through interviewing older women who are from visible minorities and/or language minorities, living in rural or urban areas, and, in addition, interviewing those who, in a paid or unpaid capacity, provide support to women experiencing IPV. In this paper, we focus on Black Nova Scotian women's experiences of IPV in Nova Scotia.

\section{Geographic Context}

Results of the 2016 Canada Census show that 37\% of the people who are visible minorities in Nova Scotia are Black (Statistics Canada 2016). About $80.7 \%$ of African Nova Scotians were born in the province, while 6.7\% were born elsewhere in Canada (African Nova Scotian Affairs 2019). Nova Scotia has ten Black communities that surround the capital city of Halifax, and roughly $75 \%$ of the African Nova Scotian population are Canadians of three or more generations (African Nova Scotian Affairs 2019). Nevertheless, Black people in Nova Scotia continue to experience racism (Bundy 2019), from the forced displacement of Africville residents in the 1960s, the racial divides of the 1989 Cole Harbour High School brawls, to the 2019 Human Right Commission's report on disproportionate street checks on Black Nova Scotians. This racism continues to be present and pervasive in Nova Scotia.

\section{Participants and Participant Recruit- ment}

We identified potential participants in several ways. We created and distributed a poster about the study, placing it on public bulletin boards (e.g., libraries, health centres) and through social media (e.g., websites, Facebook, Twitter). Information about the study and the poster were distributed through organizations identified as service providers to women experiencing IPV. Inclusion criteria consisted of women who self-identify as being older, who had experienced intimate partner violence in the past, and who identified as Black. Inclusion criteria for supporters consisted of anyone who had provided paid or unpaid support to an older Black woman experiencing IPV. We also invited those who participated to share information about the study to other potential participants. In all cases, interested participants contacted the researchers directly to have any questions about the study answered, complete the informed consent process, and to organize the time and place for the interview, or to arrange a telephone interview.

While we undertook these various strategies to recruit participants, we were only able to recruit four participants. The challenges we experienced must be contextualized within a general reluctance by Black women to come forward and share their experiences about IPV and emotional issues in general, particularly when they are asked to do this for university research. We collected indepth interview data from a Black woman who identified as being in the target age-group who had experienced IPV in the past, and three people who provide support in a paid capacity to Black women. We received approval for this research from the Research Ethics Boards at Dalhousie University (\#2018-4433), the University of New Brunswick (2018-007), and the Université de Moncton (\#1718-070).

\section{Procedures}

The semi-structured interview guide for women who have experienced IPV included questions about their definition of abuse, their personal experiences of IPV, their knowledge about services or supports, the supports and services utilized in leaving an abusive partner, and challenges they experienced in utilizing services. In addition, they were asked to give their recommendations for changes to better support women who experience IPV. The semi-structured interview guide for supporters of women who experience IPV included questions about their role, their experiences providing support, the unique needs of these women, and the strengths and challenges in supporting women. In addition, they were asked for recommendations for changes to better support women. 
Before beginning, the interviewer reviewed the information in the informed consent form and answered any questions. A signed informed consent form was received from each participant prior to the interview. Participants consented to anonymous quotes being included in any reports of the results and that the province where the study was conducted would be disclosed. All participants interviewed received an honorarium of $\$ 25$. The interviews were conducted from December 2018 to March 2019 and ranged in length from twenty-nine to fortytwo minutes. Two were conducted by phone and two were conducted face-to-face. The interviews were audiorecorded and transcribed verbatim.

\section{Data Analysis}

An interpretive narrative approach to data analysis was utilized (Polkinghorne 1988; 1995). This approach is inductive in nature, allowing research findings to emerge from the frequent, dominant, or significant narrative themes inherent in the raw data. Themes typically reflect the questions posed during an interview or focus group and, ultimately, reflect the research objectives and questions that were used to develop the interview and focus group questions.

This approach recognizes the oral tradition of people of African descent, in which storytelling is a key feature. The interpretive narrative approach focuses on highlighting direct quotes and statements voiced by participants, enabling them to articulate and give meaning to their experiences in their own words. Creating and identifying meaning within these stories empowers participants to directly represent themselves while challenging Western notions that narratives and oral storytelling are not empirical, objective, or valid. The interpretive narrative approach, therefore, recognizes humans as self-interpreting beings whose understanding of phenomena is embodied in social, cultural, and linguistic practices. Our interpretation of the data also involved ensuring that an intersectional analytical framework was used to examine how Black women's experiences of and help-seeking for IPV were influenced by the intersections of race, culture, gender identity, age, income, and socio-economic status.

Several steps were taken to analyse the data. First, the transcripts were read, and themes were identified based on the questions in the interview guides and on the study objective. The study objective was to gain insights into the needs of diverse older women who experience IPV through interviewing women from visible and lan- guage minorities and those who support them. The themes were used to create headings under which statements or direct quotes (narratives) from participants were transcribed. This process was repeated until all relevant quotes related to the themes were included under each heading. The researcher then interpreted and briefly summarized participants' quotes in each section before presenting the quotes as stated by the participant (please see the study results in the next section). This analytical process established clear links between the research objective, the interview questions, and the findings derived from the raw data. An interpretive narrative approach encourages the researcher to attend to the ways that participants construct and express their perspective of social relations. Its goal is to examine participants' multiple truths, rather than to confirm any hypothesis.

Clear links were established between the research objective and the findings derived from the raw data. In the next section of this paper, we present our findings organized by the themes identified.

\section{Results}

In this section, we report results from interviews from four participants, identifying each by a pseudonym: a Black woman who experienced IPV in the past (Jane); a Black nurse (Wanda); and two representatives of service agencies that provided support to women (Tina and Sue). Our data analysis process resulted in the identification of five themes: descriptions of the experiences if IPV for Black women; strategies for coping with IPV; strategies in supporting Black women experiencing IPV; barriers in accessing supports; and challenges in service delivery. We present a narrative description of each theme along with illustrative quotes.

\section{Experiences of IPV for Black Women}

IPV is endemic in any Black community that continues to deal with intergenerational trauma stemming from historical, structural, and institutional inequalities. Wanda discussed how a lack of opportunities for Black men often leads them to take their frustrations out on their partners:

It's like dominos. Black males...no self-esteem, no self-worth, unable to get work in their chosen profession or their line of work. Starts doing other things like either selling alcohol, selling drugs. End up with a 
partner, you know, or a wife. Angry with the partner. Starts beating, degrading, you know, just putting this person down because they don't feel good about themselves. It's like a vicious circle.

The code of silence for Black women experiencing abuse was pervasive. Wanda discussed the code of silence around IPV in her community:

It's quite an issue in our community because one of the things I've found, that a lot of Black women, especially older women, are very quiet about...they suffer in silence an awful lot. So that's been quite a challenge trying to get them to speak about it where they feel safe and they don't feel that they're going to be judged or penalized in any way.

Tina observed that many African Nova Scotian women who experience IPV often mask their depression in specific ways:

When we see African Nova Scotian women who have experienced IPV, we usually see a depressed state first that is very uniquely masked, in that, although these women are depressed, they carry themselves as if there's not a care in the world.

\section{Strategies for Coping with IPV}

Jane shared that while she has never sought support from women's centres or other resources to help her deal with her experiences of IPV, she coped with her experiences in other ways, including relying on her belief in God and helping others:

I put myself in Jesus and that's what kept me going, and working in the church, getting involved and doing a lot of things to live, that kept me...I get through with my frustrations by helping others. And, doing for the community. As long as I was busy doing for others, and seeing the results and the successes, it brought me a long way.

While Jane acknowledged that there are a variety of services to support women, she believed that experiences of discrimination, resulting in a lack of trust in systems which are set up to support communities, has contributed to Black people's general reluctance to share their experiences with service providers: As Jane observed, a history of oppression in the African Nova Scotian community, as well as a general mistrust of service providers have contributed to non-disclosure and a code of silence among Black women who have experienced IPV. In addition, Black people have traditionally relied on religion, faith, and church to cope with the challenges they face. While Tina shared similar sentiments about church, family, and friends being the main sources of support for African Nova Scotian women who experience IPV, she also identified praying, humming, and singing as three coping mechanisms that African Nova Scotian women use that she believes are unique to their culture:

African-Nova Scotian women whose family don't know that they were victims of IPV keep very quiet about it. They pray a lot. Church is a big strength, a piece of strength... a lot of African-Nova Scotian women, there's a thing that they hum and sing. Those are also ways of coping.

\section{Strategies in Supporting Black Women Experien- cing IPV}

While there were many factors contributing to Black women not seeking supports for IPV, the participants discussed instances in which they had worked with Black women. Wanda noted that many African Nova Scotian women who have experienced IPV are considerably resourceful and know where to receive support and share that information with other women:

What I've found to be true is that their resourcefulness is amazing. They know where to go to receive the assistance that they need. And what they do is they build the capacity of other women in the community by sharing that information that they've received.

In her efforts to support Black women who have experienced IPV, Sue shared that asking the right questions can often be helpful in identifying the kinds of supports and resources these women and their children need: "Who are you as a person? What makes up your support circle? How can I best understand who you are so that I can help you along this journey?"

Wanda also discussed how she supports Black women who experience IPV. She provides services in a very individualized way, and she pays particular attention to both what the women say and do not say: "I listen to their story. I hear, I take their history, hear what they have to 
say... and sometimes it's not even exactly what they're saying but reading between the lines, looking at their gestures." Tina also discussed how their service supports Black women who experience IPV with individualized and culturally appropriate options:

So, we have done women circles where it gives an opportunity for them to share resources that have helped them. We also have had kitchen table talks where we have talked specifically about being a survivor, what that looks like, provide some peer support. Even provide some rituals around how we can...from the African Nova Scotian community, how we can utilize those rituals as a tool for healing.

Tina felt that Black women feel comfortable accessing their services because she is an African Nova Scotian woman who has experienced IPV herself and because the support worker, who is White, has experience working with African Nova Scotians:

The other strength is that our women support worker...although she's of Eurocentric background, she has some experience within the African Nova Scotian community working with the Black Educators Association and what have you. So, she comes with a bit of a competency in working with African Nova Scotians.

\section{Barriers to Accessing Supports}

Our results showed that Black women experience several barriers to accessing supports for IPV, including lack of education about IPV, lack of information and awareness about available services that can support them, lack of trust in healthcare and social service professionals, reluctance to further stigmatize Black men and the Black community by disclosing experiences of IPV, fear of police involvement given the tense relationship between the Black community and the police, and a lack of restorative justice approaches. Jane noted that although Black women have traditionally lacked awareness about available services for IPV, they are becoming more educated about different types of abuse and more aware of services that continue to become more available. Racist and stigmatizing perceptions of the Black community, as well as the unavailability of services that can intervene in ways that don't cause further harm to the Black community, contributed to the barriers Black women face in accessing services. Sue pointed out, "Black women spoke about feeling concerned about leaving an impression about the Black community and violence that would then not be positive. They spoke about that as being one of the barriers to accessing services."

Sue discussed the challenges Black women encounter seeking informal and formal supports in rural communities, where there is a lack of anonymity and privacy, as well as a fear of judgement if you are Black.

Not wanting someone to judge you or your partner based on that experience. So, that can be a real challenge particularly in a rural community. And when you link that into experiences of systemic racism where you're feeling, because of the colour of your skin, that you're potentially being judged as being from a community where there's more violence, then that becomes really complicated.

Wanda also identified lack of trust in agencies and organizations, as well as their failure to hire Black staff as key reasons why Black women in and beyond midlife who experience IPV fail to access shelters and other supports.

If a Black woman is in an abusive relationship in her home, unless she has family support, many Black women do not go to the shelters. Many that I've known. And particularly the older women, they will not. That's foreign for them. That's not something that they have been used to ... And so, what happens is that there's not a lot of trust in agencies and organizations. And particularly because they're not... most of the time, they're not staffed with Black people.

Wanda also indicated that the lack of available services that cater to Black women, as well as stigma, shame, embarrassment, and fear of sharing their experiences with IPV are other factors that contribute to a general hesitancy among Black women to access services after leaving their partner or being kicked out. Tina echoed sentiments expressed by Wanda that the lack of Black service providers, as well as a lack of cultural competency demonstrated by some organizations also create barriers to seeking help. Low income, lack of access to transportation, fear of racism from service providers, language barriers, and a lack of understanding of how IPV impacts their well-being are factors that also deter older 
Black women from accessing support services, according to Tina.

A lot of our African Nova Scotian women, even the older women, a lot of us are on limited income. So, having access to a vehicle to get into town. And for fear of what may await them, the trust that may not be there, the racism that may be there. Because that's something that no matter where we go, it's always in the back of your mind.

\section{Addressing Challenges to Service Deliv- ery}

Barriers in accessing services and gaps in service delivery can be addressed in several ways, including developing partnerships with Black community groups, churches, and organizations to conduct outreach to Black women, creating initiatives or campaigns to decrease stigma around IPV in the Black community, conducting workshops and disseminating information on social media and through Black churches and organizations to raise awareness about IPV and available services, and providing mental health outreach services and other support services within Black communities. In addition, creating a service that brings together Black counsellors to provide support to Black women experiencing IPV would help address some of their mental health concerns. According to Wanda, "What would be great is if we had a group of Black counsellors for women."

A central component of cultural competency is ensuring that staff and service providers reflect the population they serve. This means that greater efforts must be made to hire racially and culturally diverse support workers with diverse experiences and skills.

Cultivating partnerships and better relationships with the Black community can also help organizations gain better insight into the specific challenges Black women face in their communities. The agencies can be better equipped to identify signs of abuse and support Black women when they are taking the necessary steps to leave abusive relationships.

Wanda pointed out that having an understanding of older Black women's unique experiences historically is a crucial aspect of culturally competent training that support workers must have.
People who understand the elder population—elder Black woman population-you know, who have an understanding, who have some insight, and who can relate to the community concerns. What's this community about? You know, what's happened here historically?

Wanda also observed that the heterogeneity of the Black population requires that support workers understand the unique needs of culturally diverse Black populations in Nova Scotia:

And there are cultural differences within the, I'll say, indigenous African population or African Nova Scotian people who have been born and raised here, and the immigrant African population. You know, there are cultural differences. There are many similarities but there are many cultural differences. So, I think that needs to be acknowledged.

Sue noted that support workers must also have an understanding of the role that systemic racism plays in Black women's lives-something she came to realize in her own work supporting Black women.

\section{Discussion}

For Black women whose experiences reside at the intersection of state-sanctioned racial and gendered violence and violence in the home, as discussed previously in this paper, IPV advocacy may not be as simple as it is for White women because it requires that they pit themselves against their men and, consequently, the Black community, which continues to be subjected to ongoing stigmatization based on enduring racist ideologies. Therefore, many Black women prefer to stay silent about their experiences with IPV to challenge ongoing forms of racism that they and their community continue to confront in all sectors of society.

In this paper, we identify several factors that deter Black Nova Scotian women in and beyond midlife from accessing support services for IPV in Nova Scotia. An important contribution of this study is our intersectional approach that contributes to our scant knowledge of Black women in and beyond midlife who experience IPV (McGarry, Simpson and Hinchliff-Smith 2011; Rezazadeh and Hoover 2018). In addition to the many 
barriers that younger women experience, older Black women experiencing IPV may be even less likely to seek help from formal service providers. Our results showed that similar to younger Black women, Black women who are in and beyond midlife utilize faith and religion to cope with IPV (St Vil et al, 2017), but we identified that praying, humming, and singing were specific coping strategies.

Our results suggest that support services must be prepared to support Black women in and beyond midlife in holistic ways that acknowledge how all forms of traumatic adverse life events and existing traumas can impact Black women's health and their ability to access services (Long and Ullman 2016; Sabri et al. 2016). They must also be equipped to recognize the culturally specific ways in which Black women present symptoms of distress. Moreover, there is an increasing cultural diversity in the Black community in Nova Scotia as the historical African Nova Scotian community lives alongside a growing newcomer population comprised of people from various African countries and, to a lesser extent, the Caribbean. Thus, support services need to appreciate the implications of this heterogeneity in how they conduct outreach to diverse Black communities and bring training programs that develop service providers' competencies in understanding and addressing the needs of culturally diverse Black communities. Black women have shared and unique experiences of trauma that influence their propensity to seek help for IPV, including a legacy of racial oppression, in the case of African Nova Scotians, and war and political persecution, in the case of some African immigrants.

A trauma-informed approach to care can be useful in centering racism as an important aspect of providing support to Black and other racialized women and communities. This is a strength-based approach that understands and responds to the impact of trauma on individuals and communities, and it emphasizes physical, psychological, and emotional safety for patients, health professionals, and other support workers (Hopper, Bassuk \& Olivet 2010). Being trauma informed is about acknowledging trauma, building welcoming and safe physical and emotional environments, promoting safety, trust, and respect in our daily interactions, and ensuring positive social interactions with clients, families, staff, volunteers, and physicians. It is also about being clientcentered, valuing collaboration and partnerships, having choices and the freedom to make choices, and recognizing client empowerment (Wisconsin Department of
Health Services 2018).

Support services for women who have experienced IPV must facilitate outreach to Black women in and beyond midlife who may not want to access existing community supports. This type of approach allows the organization to address their clients' needs by partnering with an interprofessional team of service and health professionals, Support services must also be based in transformative justice principles that challenge White, middle-class feminist understandings of IPV and approaches to seeking justice. These understandings and approaches do not often appreciate the larger historical and structural contexts that shape how Black women seek help for and cope with experiences of IPV, and that create barriers and opportunities for them to reach out for support or seek redress. In other words, without an analysis that speaks to the realities of Black women's lives, support services will fail to appreciate the specific ways in which Black women conceptualize and seek help for IPV (Bierra et al. 2006).

Participants in this study shared a similar sentiment regarding the need for more services to be made available that enable Black women in and beyond midlife to see themselves, either in the service providers hired, or in the acknowledgement of their unique experiences as Black women in the delivery of support services. Our results point to the importance of hiring support workers who reflect the racial and cultural backgrounds of the broader community, including older women. Etowa, Price, \& Debs-Ivall (2011) observe that diversity in the healthcare workforce can help address health inequities experienced by racialized and ethnic minority communities, as well as provide culturally sensitive care to all Canadians. Similarly, Pacquiao (2007) found that the advantages of increased diversity in the healthcare workforce include the fact that minority professionals are more likely to serve minority and medically underserved populations.

We drew our results from four interviews conducted in one Canadian province. Our small sample size could be considered a limitation as we were not able to represent the diversity of older Black women's experiences. While we gained insights in this study, the results should not be generalized to other locations and cultural groups. Additional research is needed to further illuminate the specific barriers and recommendations for change for Black women within other geographic and political contexts (Lacey et al. 2015). However, our research does provide important insights about the experiences, barriers faced, 
and how to address these challenges for Black women who experience IPV in and beyond midlife. It is clear that IPV within Black communities can only be effectively addressed if support services are prepared to help dismantle the systems of oppression that continue to harm Black communities and to address the intersecting forms of violence that Black women experience through both state agencies and intimate partnerships.

\section{Works Cited}

African Nova Scotian Affairs. 2019. African Nova Scotian Community. https://ansa.novascotia.ca/community.

Alimahomed-Wilson, Jake, and Dana Williams. 2016. "State Violence, Social Control, and Resistance." Journal of Social Justice 6: 1-15.

Al'uqdah, Shareefah, Casilda Maxwell, and Nicholle Hill. 2016. "Intimate Partner Violence in the African American Community: Risk, Theory, and Interventions." Journal of Family Violence 31, no. 7: 877-84. doi: 10.1007/s10896-016-9819-x.

Bent-Goodley, Tricia B. 2013. "Domestic Violence Fatality Reviews and the African American Community." Homicide Studies 17, no 4: 375-390. doi: 10.1177/1088767913497949.

Bierra, Alisa, Onion Carrillo, Eboni Colbert, Xandra Ibarra, Theryn Kigvamasud'Vashti, and Shale Maulana. 2006. "Taking Risks: Implementing Grassroots Community Accountability Strategies." In Color of Violence: The Incite! Anthology, edited by The INCITE! Women of Color Against Violence, 250-266. Cambridge, Mass.: South End Press.

Bonomi, Amy E, Melissa L Anderson, Robert J Reid, David Carrell, Paul A Fishman, Frederick P Rivara, and Robert S Thompson. 2007. "Intimate Partner Violence in Older Women." The Gerontologist 47, no. 1: 34-41. doi:10.1093/geront/47.1.34.

Bourget, Dominique, Pierre Gagné, and Laurie Whitehurst. 2010. "Domestic Homicide and Homicide-Suicide: The Older Offender." The Journal of the American Academy of Psychiatry and the Law 38, no. 3: 305-11.

Bukowski, Leigh, A., Melvin C. Hampton, Cesar G. Escobar-Viera, Jordan M. Sang, Christian J. Chandler, Emmett Henderson, Stephanie L. Creasy, and Ronald D. Stall 2019. "Intimate partner violence and depression among Black transgender women in the USA: The po- tential suppressive effect of perceived social support". Journal of Urban Health 96, no. 5: 760-771. 10.1007/ s11524-019-00355-3

Bundy, Jessica. 2019. "'We'll Deal with it Later': African Nova Scotian Women's Perceptions and Experiences of the Police." Canadian Journal of Sociology 44, no. 4: 31941.

Etowa, Josephine B, Brenda L Beagan, Felicia Eghan, and Wanda Thomas Bernard. 2017. "You Feel You Have to Be Made of Steel": The Strong Black Woman, Health, and Well-being in Nova Scotia." Health Care for Women International 38, no. 4: 379-393. doi: 10.1080/07399332.2017.1290099.

Etowa, Josephine, Sheri Price, and Salma Debs-Ivall. 2011. "Strengthening the Ethno-Cultural Diversity of the Nursing Workforce in Canada." International Journal of Arts \& Sciences 4, no. 26: 75-87.

Feagin, Joe R. 2000. Racist America: Roots, Current Realities, and Future Reparations. ( ${ }^{\text {rd }}$ Edition). New York: Routledge.

Gazso, Amber, Susan McDaniel, and Ingrid Waldron. 2016. "Networks of Social Support to Manage Poverty: More Changeable than Durable." Journal of Poverty 20, no. 4: 441-63. doi: 10.1080/10875549.2015.1112869.

Gazso, Amber, and Ingrid Waldron. 2009. "Fleshing Out the Racial Undertones of Poverty for Canadian Women and Their Families: Re-envisioning a Critical Integrative Approach." Atlantis 34, no. 1: 132-141.

Godoy-Ruiz, Paula, Brenda Toner, Robin Mason, Carolina Vidal, and Kwame McKenzie. 2015. "Intimate Partner Violence and Depression Among Latin American Women in Toronto." Journal of Immigrant and Minority Health 17, no. 6: 1771-1780. doi: 10.1007/s10903-0140145-1.

Hicken, Margaret, Nicole Kravitz-Wirtz, Myles Durkee, and James Jackson. 2018. "Racial Inequalities in Health: Framing Future Research." Social Science \& Medicine 199: 11. doi: doi.org / 10.1016/.socscimed.2017.12.027.

Hightower, Jill, M.J. Smith, and Henry C. Hightower. (2006) "Hearing the Voices of Abused Older Women." Journal of Gerontological Social Work 46, no. 3-4: 205-227. doi:10.1300/J083v46n03_12.

hooks, bell. 1993. Sisters of the Yam: Black Women and Self-recovery. Boston, MA: South End Press. 
Hopper, Elizabeth K., Ellen L. Bassuk, and Jeffrey Olivet. 2010. "Shelter from the Storm: Trauma-Informed Care in Homelessness Services Settings." The Open Health Services and Policy Journal 3: 80-100.

Lacey, Krim K, Karen Powell Sears, Niki Matusko, and James S Jackson. 2015. "Severe Physical Violence and Black Women's Health and Well-being." American Journal of Public Health 105, no. 4: 719-724. doi: 10.2105/AJPH.2014.301886.

Long, LaDonna, and Sarah E Ullman. 2016. "Correlates of Problem Drinking and Drug Use in Black Sexual Assault Victims." Violence and Victims 31, no. 1: 71-84. doi: 10.1891/0886-6708.VV-D-14-00024.

Martin, Agnes, Noreen Boadi, Caroline Fernandes, Sherry Watt, and Tracy Robinson-Wood, 2013. "Applying resistance theory to depression in Black women," Journal of Systemic Therapies 32, no 1: 1-13. https:// doi.org/10.1521/jsyt.2013.32.1.1.

Mcgarry, Julie, Chris Simpson, and Kathryn HinchliffSmith. 2011. "The Impact of Domestic Abuse for Older Women: A Review of the Literature." Health \& Social Care in the Community 19, no 1: 3-14. doi:10.1111/ j.1365-2524.2010.00964.x.

Menjivar, Cecilia. 2016. "A Framework for Examining violence." In Gender Through the Prism of Difference, edited by Maxine Baca Zinn, Pierrette Hondagneu-Sotelo, Michael A. Messner, and Amy M. Denissen, 130-145. New York: Oxford University Press.

Miles, Robert. 1989. Racism. New York: Routledge.

Montero, Isabel, David Martín-Baena, Vicenta EscribàAgüir, Isabel Ruiz-Pérez, Carmen Yives-Cases, and Marta Talavera. 2013. "Intimate Partner Violence in Older Women in Spain: Prevalence, Health Consequences, and Service Utilization." Journal of Women \& Aging 25, no 4: 358-371. doi:10.1080/08952841.2013.83885.

Okeke-Ihejirika, Philomena, Bukola Salami, and Ahmad Karimi 2019. “African Immigrant Women's Transition and Integration into Canadian Society: Expectations, Stressors, and Tensions." Gender, Place \& Culture 26, no 4: 581-601. https://doi.org/ 10.1080/0966369X.2018.1553852.

Pacquiao, Dula. 2007. "The Relationship Between Cultural Competence Education and Increasing Diversity in Nursing Schools and Practice Settings." Journal of
Transcultural Nursing 18, no. 1: 28S-37S. doi : $10.1177 / 1043659606295679$.

Pathak, Neha, Rageshri Dhairyawan, and Shema Tariq. 2019. "The Experience of Intimate Partner Violence among Older Women: A Narrative Review." Maturitas 121: 63-75. doi: 10.1016/j.maturitas.2018.12.011.

Pellow, David N. 2016. "Toward a Critical Environmental Justice Studies: Black Lives Matter as an Environmental Justice Challenge.” Du Bois Review 13, no 2: 116.

Polkinghorne, Donald E. 1988. Narrative Knowing and the Human Sciences. Albany: State University of New York Press.

Polkinghorne, Donald E. 1995. "Narrative configuration in qualitative analysis." International Journal of Qualitative Studies in Education 8, no 1: 5-23. doi:

$10.1080 / 0951839950080103$

Rezazadeh, Maryam S., and Michael L. Hoover. 2018. "Women's Experiences of Immigration to Canada: A Review of the Literature." Canadian Psychology 59, no. 1: 76-88. doi:10.1037/cap0000126.

Roberto, Karen A., Marya C. McPherson, and Nancy Brossoie. 2013. "Intimate Partner Violence in Late Life." Violence Against Women 19, vol 12: 1538-1558. doi:10.1177/1077801213517564.

Sabri, Bushra, Jamila K. Stockman, Jacquelyn C. Campbell, Sharon O'Brien, Doris Campbell, Gloria B. Callwood, Desiree Bertrand, Lorna W. Sutton, and Greta Hart-Hyndman. 2014. "Factors Associated with Increased Risk for Lethal Violence in Intimate Partner Relationships among Ethnically Diverse Black Women." Violence and Victims 29, no. 5: 719-41. doi: 10.1891/0886-6708.VV-D-13-00018.

Sabri, Bushra, Julia Huerta, Kamila A. Alexander, Noelle M. St. Vil, Jacquelyn C. Campbell Gloria B. Callwood. 2015. "Multiple Intimate Partner Violence Experiences: Knowledge, Access, Utilization and Barriers to Utilization of Resources by Women of the African Diaspora." Journal of Health Care for the Poor and Underserved 26, no 4: 1286-1303. doi:10.1353/hpu.2015.0135.

Sabri, Bushra, Charvonne N Holliday, Kamila A Alexander, Julia Huerta, Andrea Cimino, Gloria B Callwood, and Jacquelyn C Campbell. 2016. "Cumulative Violence Exposures: Black Women's Responses and Sources of 
Strength." Social Work in Public Health 31, no. 3: 127 139. doi: 10.1080/19371918.2015.1087917.

Schreiber, Rita, Phyllis Noerager Stern, and Charmaine Wilson. 2000. "Being Strong: How Black West-Indian Canadian Women Manage Depression and Its

Stigma." Journal of Nursing Scholarship 32, no. 1: 39-45. doi:10.1111/j.1547-5069.2000.00039.x.

Sormanti, Mary, and Tazuko Shibusawa. 2008. "Intimate Partner Violence among Midlife and Older Women: A Descriptive Analysis of Women Seeking Medical Services." Health \& Social Work 33, no. 1: 33-41.

Statistics Canada. 2016. "Immigration and Ethnocultural Diversity Highlight Tables.” November 13, 2020. https://www12.statcan.gc.ca/census-recensement/2016/ dp-pd/hlt-fst/imm/index-eng.cfm.

Stöckl, Heidi, and Bridget Penhale. 2015. "Intimate Partner Violence and Its Association with Physical and Mental Health Symptoms Among Older Women in Germany." Journal of Interpersonal Violence 30, no. 17: 3089-111. doi:10.1177/0886260514554427.

St Vil, Noelle M, Bushra Sabri, Vania Nwokolo, Kamila A Alexander, and Jacquelyn C Campbell. 2017. "A Qualitative Study of Survival Strategies Used by Low-Income Black Women Who Experience Intimate Partner Violence." Social Work 62, no. 1: 63-71. doi: 10.1093/ sw/sww080.

Tam, Dora, M. Y., Leslie M. Tutty, Ze Hong Zhuang, and Eva Paz. 2016. "Racial Minority Women and Criminal Justice Responses to Domestic Violence." Journal of Family Violence 31, no. 4: 527-38. 10.1007/s10896015-9794-7.

Waldron, Ingrid R. G. 2019. “Archetypes of Black womanhood: Implications for Mental Health, Coping and Help-Seeking." In Culture, Diversity \& Mental Health: Enhancing Clinical Practice, edited by Masood Zangeneh and Alean Al-Krenawi, 21-38. New York: Springer

Nature.

Waldron, Ingrid R. G. 2002. African Canadian Women Storming the Barricades!: Challenging Psychiatric Imperialism through Indigenous Conceptualizations of "Mental Illness" and Self Healing. Unpublished doctoral thesis, University of Toronto.
Waldron, Ingrid, and Amber Gazso. 2017. "Managing Low Income in Families: The Importance of Institutions and Interactions." In Continuity and Innovation: Canadian Families in the New Millennium, edited by Amber Gazso \& Karen Kobayashi, 80-96. Toronto: Nelson Education.

Weeks, Lori E, and Kristal Leblanc. 2011. “An Ecological Synthesis of Research on Older Women's Experiences of Intimate Partner Violence." Journal of Women and Aging 23, vol 4: 283-304. doi:10.1080/08952841.2011.611043.

Weeks, Lori. E., Christie Stilwell, Danie Gagnon, Suzanne Dupuis-Blanchard, Colleen MacQuarrie, and Lois Jackson. 2021. "Initiatives to support older women who experience intimate partner violence." Violence Against Women advance online publication. doi.org/ $10.1177 / 1077801220988355$.

Wisconsin Department of Health Services. 2018. "Trauma-informed care principles," Wisconsin Department of Health Services. https://www.dhs.wisconsin.gov/tic/principles.htm. 